\title{
Hyperdiploidy Tetraploidization and Genomic Instability in Breast Cancer - A Case Report Study
}

\section{Roland B Sennerstam ${ }^{1 *}$ and Jan-Olov Strömberg ${ }^{2}$}

${ }^{1}$ Department of Pathology and Oncology Karolinska Hospital and Karolinska Institutet SE-171 76 Stockholm, Sweden ${ }^{2}$ Department of Mathematics, Royal Institute of Technology, SE-100 74 Stockholm, Sweden

\begin{abstract}
Introduction: Several reports have indicated that tetraploidization is an intermediate step of tumor progression between diploid cells and genomically reorganized aneuploid tumor cells. Tetraploidization is a conserved phenomenon in both plants and animals, which occurs in the human body in reaction to various stress factors.

Methods: A breast cancer population was divided into groups according to DNA Index (DI) interval, and three ploidy entities were defined as three different tumor groups: diploid (D-type), tetraploid (T-type) and aneuploid (A-type) tumors. Using a parameter reflecting genomic instability and proliferative activity (Stemline Scatter Index, SSI), we stepwise simulated the ploidy alterations following increase in SSI values. The percentage of each tumor type at each level of accumulated SSI value was estimated and the slopes of the generated curves were compared in linear regression analysis.
\end{abstract}

Results: At diagnosis, $32 \%$ of the patients had T-type tumors some of which were established in a pre-diagnostic period. During simulation guided by increasing SSI values a second step of tetraploidization was found during a tumor size interval of $10-20 \mathrm{~mm}$ caused by a presumed reaction to anoxic stress. The generated tetraploid cell populations were recruited from diploid cancer cells, indicating that already transformed cells loaded tetraploidgenerated cells with genomic instability. These genomically unstable and altered tetraploid cells were postulated to generate aneuploid cells within a hypotetraploid DI region. A narrow DI interval among D-type tumors was suggested to be responsible for the recruitment of T-type tumors.

Conclusion: We present a two-step model in which the first tetraploidization occurs early in breast cancer tumor progression and might represent a reaction to stress factors in benign epithelial tumors and epithelial hyperplasia and a later process of tumor size dependent origin for tetraploidization.

Keywords: Genomic instability; Tumor progression; Tetraploidization; Breast cancer

\begin{abstract}
Abbreviations: D-type tumors- Diploid Tumors; T-type tumors: Tetraploid Tumors; A-type tumors: Aneuploid Tumors; DI: DNAIndex; SSI: Stemline-Scatter-Index; G1-phase: Gap1 Phase before SPF; G1CV: Coefficient of Variation of G1 Peak; SPF: S-Phase Fraction; G2phase: Gap 2 after SPF; Exc-G2: Cells of DNA-Values above DI $>2.2$; PI: Proliferation Index; BC: Breast Cancer; VEGF: Vascular Endothelial Growth Factors
\end{abstract}

\section{Introduction}

Polyploidization is a well-known physiological phenomenon that has been observed in plants, amphibians, fish and yeast [1]. It can occur as endoreplication, i.e. the completion of two rounds of DNA-replication without intermediate mitosis generating tetraploid cell nuclei [2], after karyokinesis without subsequent cytokinesis generating binucleated cells, cell-to-cell fusion as in myocytes [3], or can be generated by virus fusogenic proteins [4]. An elevated level of tetraploidy was previously reported in women diagnosed by the Papanicolaou smear as Atypical Squamous Cells of Undetermined Significance (ASCUS) and in combination with Human Papilloma virus (HPV)-positive cells [5], and might represent a conserved reaction to stress caused by the HPV infection or by fusogenic proteins.

During carcinogenesis, tetraploidization has been suggested to be an intermediate stage towards aneuploidy [6-12]. After mitotic disruption and failed daughter cell segregation, the cells enter into another G1-phase generating a tetraploid population with increased genomic instability. Transgenic mice expressing an SV40 T-antigen under the control of the rat regulatory elements from the rat elastase
I gene reproducibly developed pancreatic tumors from acinar cells [13]. Cancer development occurred in a stepwise model from diploid $\rightarrow$ tetraploid $\rightarrow$ aneuploidy cells, corresponding to a sequence of normal acinar cells to hyperplasia to dysplasia and ultimately to invasive cancer. This study is of particular interest because diagnosis of pancreatic cancer frequently identifies aneuploid tumors because the tumor is first apparent when liver metastasis is established.

Tetraploidization has been observed because of short-term hypoxia in developing breast cancer in transgenic mice [14] and [15]. Short hypoxic periods $(24 \mathrm{~h})$ in tissue culture of the human hyperdiploid melanoma cell line BEX-c resulted in an increased level of endoreplication. After another $24 \mathrm{~h}$ of hypoxia, the population homogenized as tetraploid cells [16]. Furthermore, the effect of oxidative stress in human breast cancer has been reported to be associated with increased genomic instability [17-21]. The origin of this sort of stress might occur after obtaining a certain tumor size resulting in a lack of oxygen and nutrition in the centre of tumor mass. A solution to this

*Corresponding author: Roland B Sennerstam, Department of Pathology and Oncology Karolinska Hospital and Karolinska Institutet SE-171 76 Stockholm, Sweden, Fax: 0046-8-331-696; E-mail: roland.sennerstam@ki.se

Received May 09, 2013; Accepted June 10, 2013; Published June 17, 2013

Citation: Sennerstam RB, Strömberg JO (2013) Hyperdiploidy Tetraploidization and Genomic Instability in Breast Cancer-A Case Report Study. J Carcinogene Mutagene 4: 144. doi:10.4172/2157-2518.1000144

Copyright: (C) 2013 Sennerstam RB, et al. This is an open-access article distributed under the terms of the Creative Commons Attribution License, which permits unrestricted use, distribution, and reproduction in any medium, provided the original author and source are credited. 
problem is a growth of blood vessels into the tumor mass supported by Vascular Endothelial Growth Factors (VEGF), which are needed to increase the micro-vessel density of tumors. Breast tumors rich in VEGF staining intensity was found in $14 \%$ among tumors having a size below $20 \mathrm{~mm}, 55 \%$ in those between $21-50 \mathrm{~mm}$ and $31 \%$ in those above $51 \mathrm{~mm}[22]$.

Studies of Comparative Genomic Hybridization (CGH) found that early genomic alterations during tumor growth involve many chromosome segmental gains and some losses as well as regional amplifications. Comparing breast tumors $\mathrm{T} 1 \mathrm{~b}(\leq 10 \mathrm{~mm})$ and $\mathrm{T} 1 \mathrm{c}(11-$ $20 \mathrm{~mm}$ ), a fourfold increase in regional amplifications was observed in T1c compared with T1b [23]. Studies of human cervical HPV infected condyloma all were diploid but cervical intraepithelial neoplasia (CIN I-III) CIN I revealed a 50/50\% diploid/tetraploid distribution, while an increasing amount of aneuploidy (70\%) was found in CIN II-III all of which were infected by HPV [24]. This compares with breast cancer, where polyploidization and aneuploidy have been reported to occur in epithelial hyperplasia both with and without cell atypia [25]. Moreover, aneuploidy in ulcerative colitis precedes the appearance of primary colon cancer by up to one decade [26].

In breast cancer, the P53 mutant protein was present in $6 \%$ of D-type tumors, $32 \%$ of T-type and $35 \%$ of A-type, supporting the theory that T-type tumors transfer their mutational load to A-type tumors [27]. The mutant protein was first detected in carcinoma in situ [25], raising the question of whether polyploidization precedes tumor transformation as a reaction to stress factors. Mutations in the p53 gene lead to centrosome amplification and result in multipolar spindle formation, which strongly disturbs cell division $[10,24,28]$.

During the last few decades, the one-gene-one-mutation origin of cancer development has been questioned in favor of a chromosomal rearrangement theory [29]. In this paper, we used a low level of resolution, DNA ploidy, to study a large-scale alteration of the genome during tumor growth. We used three ploidy groups of tumors and simulated the increase in genomic instability by stepwise growing Stemline-Scatter-Index values reflecting chromosomal, tumor genotypic instability, and proliferative activity.

\section{Materials and Methods}

\section{Study population}

A consecutive sample of 519 breast cancer patients was analyzed from 1991. That early year was selected since it offers more than 15 years of follow up of survival data. The population was subdivided into four groups based on DNA Index (DI) intervals: (1) diploid tumors $(0.9 \leq \mathrm{DI}<1.2)$ (D-type), in which the upper border was extended to less than 1.2 to include the hyperdiploid tumors up to that level; (2) aneuploid tumors $(1.2 \leq \mathrm{DI}<1.8)$ (A-type); (3) tetraploid tumors $(1.8$ $\leq \mathrm{DI}<2.2$ ) (T-type); and (4) a small group (DI $\geq 2.2$ ) (A-type) [30]. The D- and T-type of tumors have a DI range that justifies them being known as "near-diploid" and "near-tetraploid", respectively. However, in this study, we used the shorter terminology (D- and T-type). The aneuploid (A-type) tumor group with $\mathrm{DI}>=2.2$ was excluded in the analysis since we focused on the dynamics between D-, A- and T-type tumors with $\mathrm{DI}<2.2$.

\section{Feulgen staining}

Nuclear DNA content was measured by image cytometry of Feulgen-stained imprints or $8-\mu \mathrm{m}$ histological sections from primary breast adenocarcinoma. The same pathologist who identified single tumor cells analyzed 200 cells per slide. The materials were prepared in decreasing ethanol concentrations and were exposed to hydrolysis in $5 \mathrm{M} \mathrm{HCL}$ at $22^{\circ} \mathrm{C}$ for $60 \mathrm{~min}$. Specimens were then rinsed in distilled water and stained with Schiff's reagent at $22^{\circ} \mathrm{C}$ for $90 \mathrm{~min}$. Lymph node samples were used as an internal diploid DNA standard (2C). The cells were washed three times in sulfurous acid $\left(\mathrm{H}_{2} \mathrm{SO}_{3}\right)$ and measured by computer assisted analysis systems using an axioscope (Zeiss, BadenWürttenberg, Germany) equipped with an immersion plan-objective (40/0,95; Nikon, Tokyo, Japan) and a CCD camera (Nikon).

\section{Stemline Scatter Index (SSI)}

To create a large-scale estimation of genomic instability and proliferative activity, we used the coefficient of variation for the G1 peak (G1CV) of tumor DNA stem-lines (DI) and DNA content values in the S-Phase Fraction (SPF) for each patient, plus the percentage of cells with a DNA content above the G2 DNA level (exceeding the G2 rate, Exc-G2). Thus, the SSI includes G1CV +SPF + Exc-G2, which are all expressed in percentages. In previous reports we used an SSI cut-off value of $8.8 \%$ to differentiate between tumors representing significantly scattered DNA histograms (SSI $>8.8 \%$ ) and those with insignificantly scattered ones (SSI $\leq 8.8 \%$ ) [31]. Breast carcinomas with SSIs $\leq 8.8 \%$ were classified as genomically stable and those with SSI $>8.8 \%$ as genomically unstable. We continue to apply this limit when creating SSI intervals in the present paper. By relating the three-ploidy entities to increasing SSI values, the development of genomic heterogeneity resembled a simulation and was reflected in D-, A- and T-type tumors. The G1CV and SPF are deduced to increase before Exc-G2 cells appear, so we applied an equation from a 3D surface using xyz variables in which Exc-G2 is denoted as $\mathrm{z}: \mathrm{z}=0.0152+0.0508 \mathrm{x}+0.0506 \mathrm{y}$. Thus, G1CV (x) and $\operatorname{SPF}(y)$ contribute equally to the increase in genomic instability and proliferative activity.

\section{Lognormal distribution of parameters}

In the probability theory, a lognormal distribution is a probability distribution of a random variable whose logarithm is normally distributed. If $\mathrm{x}$ is a random variable with a normal distribution then $\mathrm{Y}$ $=\exp (\mathrm{x})$ has a lognormal distribution.

A variable might be modeled as lognormal if it can be thought of as the multiplicative product of many independent random variables, each of which is positive and none of which has a decisive influence. Growing cell populations represent samples of multiple events generating a passage through the cell cycle. The parameters investigated and presented after having been analyzed as Feulgen-stained cells on slides were the G1-, S-, G2-phase and DI values, as well as G1CV. The parameters were tested in a probability-probability plot, comparing empirical against theoretical cumulative values in a lognormal curve.

\section{Distribution of DNA indices}

We analyzed the distribution of DNA indices in histograms of four increasing values of SSI intervals: $\mathrm{SSI} \leq 8.8 ; 8.8<\mathrm{SSI} \leq 12 ; 12<\mathrm{SSI} \leq 15$ and $15<$ SSI $\leq 40$. Guided by the SSI intervals, we followed the stepwise alterations in the histograms of $\mathrm{D}$-, A-, and T-type tumors

\section{Alterations in ploidy entities related to increasing SSI values}

To detect trends of change in the relation between the ploidy entities, the SSI values were subdivided into three consecutive groups of a fairly equal number of accumulatively increasing SSI values: $\mathrm{SSI} \leq 8.8$ $(\mathrm{n}=171), 8.8<$ SSI $\leq 15(\mathrm{n}=195)$, and $13<$ SSI $\leq 40(\mathrm{n}=173)$. The overlap in the boundaries between the second and the third groups enabled a smooth transition to be achieved. For each value of SSI in the curves 
Citation: Sennerstam RB, Strömberg JO (2013) Hyperdiploidy Tetraploidization and Genomic Instability in Breast Cancer-A Case Report Study. J Carcinogene Mutagene 4: 144. doi:10.4172/2157-2518.1000144

drawn, the sum of the accumulated numerical values for D-, A-, and T-type tumors was registered. From this, we calculated the percentage for each one of the three-ploidy tumors; all three percentages summed to $100 \%$ at each point of the curves. This made it possible to determine how the ploidy entities change during stepwise increases in SSI values. An alteration in one of the ploidy entities exerts an effect on one or both of the others, so tetraploidization will be seen as a reduction in D-type tumors in favor of T-type tumors. A selective transfer to hypoT-type tumors thus increasing the A-type tumor population will reduce genomically unstable T-type tumors. When a clear tend in the slope of the curve was observed, we added in the interval $8.8<\mathrm{SSI}<=15$ the SSI values as gathered in separate intervals instead of accumulating values to obtain a higher level of resolution (Figure 4c).

\section{Hyperdiploid tumors and tetraploidization}

As some diploid tumors with a certain level of hyperdiploidy can drive the entire cell population into tetraploidy [16], we analyzed the relationship between the variations of DI in D-type tumors against $\mathrm{T}$ and A-type tumors using two-dimensional linear regression scatter plots.

\section{Statistical Analysis}

Statistical calculations were performed using the STATISTICA software package (StatSoft, Inc., Tulsa, OK, USA). Statistical significance for categorical variables was calculated using the chi square test and an independent $t$-test was used for continuous ones. Linear regression was performed for the correlation test. Statistical significance was assumed if $p<0.05$. An ethics committee at Karolinska Institutet Stockholm Sweden (2010/34-31/1) approved the study design.

\section{Results}

To perform an analysis of tetraploidization during tumor progression and increasing genomic instability, we created four DI entities: (1) diploid breast tumors (D-type, $n=230 ; 44.3 \%$ ); (2) aneuploid tumors (A-type, $\mathrm{n}=106 ; 20.4 \%$ ); (3) tetraploid tumors (T-type, $\mathrm{n}=156$; $32.2 \%$ ) (Table 1$)$; and a small group of DI $\geq 2.2(\mathrm{n}=27 ; 4.2 \%)$ was excluded from analysis as described in the Materials and Methods. Figure 1a shows the relationship between tumor size and DI for those patients whose tumor size was reported $(n=461)$. The highest level of DI heterogeneity is observed for tumor sizes between $10-20 \mathrm{~mm}$.

\section{Definition of DI intervals}

In Table 1, the DI entities are defined according to DI, tumor size, SPF, Proliferation Index (PI) and SSI, and were shown to differ significantly between each other with respect to all cytochemical parameters. A-type tumors have a significantly higher level of SPF, PI and SSI in relation to D- and T-type tumors, while T-type tumors have a significantly higher level of SPF, PI and SSI compared with D-type tumors. There is also a significant difference in tumor size between $\mathrm{D}$ and A-type tumors $(p<0.05)$ but not between T- and D-type tumors or between A- and T-type tumors.

The death and survival data in Table 2 reveal a significant difference $(p<0.02)$ in the death rate from breast cancer $(B C)$ between D-type

\begin{tabular}{|c|c|c|c|c|c|c|c|}
\hline Tumor type & D-Type & & A-Type & & T-Type & & D-Type \\
\hline Total sample 492 & $n=23044.3 \%$ & $p<0.001$ & $n=10620.4 \%$ & $p<0.05$ & $\mathrm{n}=15632.2 \%$ & $p<0.02$ & $n=23044.3 \%$ \\
\hline Size $(\mathrm{mm})$ & $18.3 \pm 13.2$ & $p<0.05$ & $21.7 \pm 14.7$ & ns & $19.5 \pm 10.8$ & ns & 18.3. \pm 13.2 \\
\hline DI & $1.04 \pm 0.05$ & $p<0.001$ & $1.58 \pm 0.15$ & $p<0.001$ & $1.98 \pm 0.11$ & $p<0.001$ & $1.04 \pm 0,05$ \\
\hline SPF $\%$ & $4,33 \pm 6,47$ & $p<0.001$ & $15,7 \pm 13,7$ & $p<0.001$ & $8,01 \pm 9,00$ & $p<0.001$ & $4.33 \pm 6,47$ \\
\hline $\mathrm{PI} \%$ & $9.91 \pm 8.48$ & $p<0.001$ & $23.7 \pm 16.2$ & $p<0.001$ & $12.6 \pm 11.8$ & $p<0.01$ & $9.91 \pm 8,48$ \\
\hline SSI rel.unit & $9,96 \pm 6,64$ & $p<0.001$ & $20,7 \pm 12,2$ & $p<0.001$ & $13,9 \pm 9,87$ & $p<0.001$ & $9,96 \pm 6,64$ \\
\hline
\end{tabular}

Table 1: Presentation of the ploidy entities related to tumor size, DI, SPF, PI and SSI. The result of cytochemical parameters as DNA-index (DI), percentages of S-phase (SPF), proliferation index (PI) and Stemline-Scatter-Index (SSI) analyzed at diagnosis and their relation to ploidy entities are shown. The difference between the three ploidy entities D-, A-, and T-type tumors are statistically significant in all parameters except tumor size only between D- and A-type tumors. The D-type tumors are placed both to the left and the right side in the Table to make all comparisons possible.
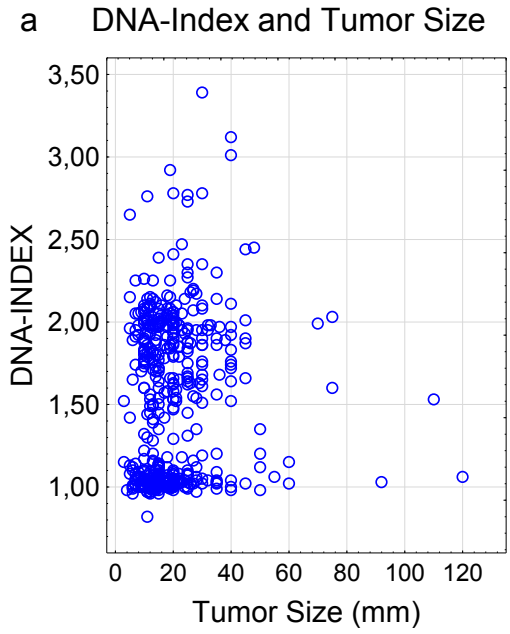

b DNA-Index and SSI

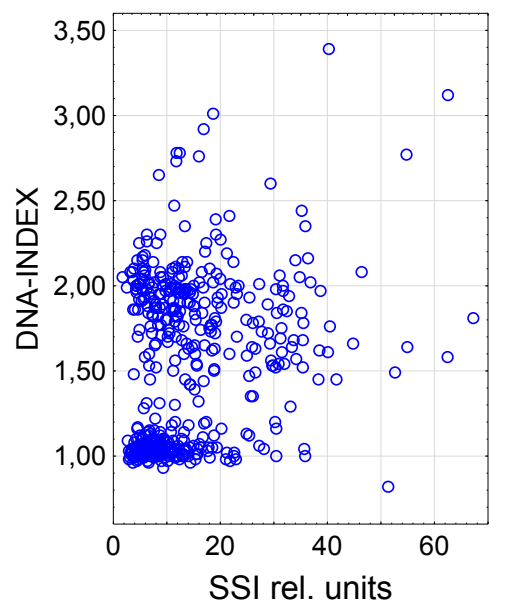

Figure 1: Relationship between DNA index (DI), tumor size and SSI. (a) The most dispersed of DI values were observed in the tumor size interval 10-20 mm. (b) The relationship between SSI and DI in the $\mathrm{DI}$ interval $1.5<\mathrm{DI}<2.2$ was shown to be significantly negative by linear regression analysis $(r=-0.32, p<0.001 ; n=222)$. 
Citation: Sennerstam RB, Strömberg JO (2013) Hyperdiploidy Tetraploidization and Genomic Instability in Breast Cancer-A Case Report Study. J Carcinogene Mutagene 4: 144. doi:10.4172/2157-2518.1000144

(23.5\% $\mathrm{n}=230)$ and A-type tumors $(36.8 \% \mathrm{n}=106)$, but no significant difference between D-type and T-type tumor $(p=0.30)$.

Since D-type tumors are associated with a longer survival time, patients with these tumors were more likely to die from reasons other than BC $(32.2 \%)$ so presented in this way there was no significant difference between $\mathrm{D}$-, $\mathrm{A}$-, and T-type tumors in survival rate (alive) after 17 years of follow-up (Table 2).

\section{Relationship between SSI and DI}

Figure $1 \mathrm{~b}$ shows the relationships between SSI and DI for the whole population. It follows a similar pattern to Figure 1a for tumor size against SSI. A linear regression analysis of the sum of T- and hypo-Type tumors in the common DI range of $1.5<\mathrm{DI}<2.2$ against SSI revealed a strong negative slope (Figure text).

\section{Lognormal probability-probability cumulative plot}

Every tumor in the sample was defined by image analysis following Feulgen staining according to G1-phase, G1CV, SPF, or G2-phase. Some tumors only had a G1 peak; others had in addition some cells in SPF, or cells in G2-phase. We regarded these data as a growing cell population fixed at a variable position in the cell cycle. The outcome was analyzed as a lognormal probability-probability distribution simulation, with the empirical cumulative curve as the $y$-axis and the theoretical cumulative curve as the $\mathrm{x}$-axis. The SPF and G2-phase closely fitted the theoretical curves (Figures $2 \mathrm{~b}$ and $2 \mathrm{c}$ ), as did the G1CV and tumor size when included (data not shown), suggesting that the population was derived from many random events. G1-values showed an initial delay in adaption to the theoretical curve and thereafter deviated to a steeper positive slope (Figure 2a). The DI sample deviated from the theoretical curve, indicating an intermediate sized alteration in DNA content (Figure 2d)(see Discussion).

\section{DNA histograms and increasing SSI values}

The DI histograms revealed significant ploidy alterations, with a significant increase in T-type tumors from $27.8 \%$ (SSI $\leq 8.8$; Figure $3 \mathrm{a}$ ) to $45.5 \%(12<\mathrm{SSI} \leq 15$; Figure $3 \mathrm{c})(p<0.02)$, which decreased thereafter to $28.1 \%$ reflecting a temporary tetraploid stage $(15<$ SSI $\leq 40$; Figure 4d) $(p<0.05)$. A-type tumors increased significantly from $9.3 \%$ to $47.1 \%$ (Figure 3a, $\mathrm{d} ; p<0.001)$. The SSI interval with the largest T-type peak was in the tumor size interval between $10-20 \mathrm{~mm}(17.5 \pm 8.5 \mathrm{~mm}$; Figure $3 \mathrm{c}$ ). The death rate in $\mathrm{BC}$ for patients in Figure $3 \mathrm{a}$ after 17 years of follow up was $28 \%$ and in Figure $3 \mathrm{~d} 41 \%(\mathrm{p}<0.02)$.

\begin{tabular}{|c|c|c|c|c|c|c|c|c|}
\hline & \multicolumn{8}{|c|}{ Tumor type } \\
\hline & \multicolumn{2}{|c|}{ D-type $0.9 \leq \mathrm{DI}<1.2$} & \multicolumn{2}{|c|}{ A-type $1.2 \leq \mathrm{DI}<1.8$} & \multicolumn{2}{|c|}{ T-type $1.8 \leq \mathrm{DI}<2.2$} & \multicolumn{2}{|c|}{ A-type DI $\geq 2.2$} \\
\hline & $\mathrm{n}$ & $\%$ & $\mathrm{n}$ & $\%$ & $\mathrm{n}$ & $\%$ & $\mathrm{n}$ & $\%$ \\
\hline Death from BC & 54 & 23.5 & 39 & 36.8 & 44 & 28.2 & 10 & 37.0 \\
\hline Death from other causes & 74 & 32.2 & 29 & 27.4 & 41 & 26.3 & 8 & 29.7 \\
\hline Alive & 102 & 44.3 & 38 & 35.8 & 71 & 45.5 & 9 & 37.3 \\
\hline Total & 230 & 100 & 106 & 100 & 156 & 100 & 27 & 100 \\
\hline
\end{tabular}

Table 2: Survival and death rates in breast cancer. Death rate in breast cancer (BC), death for other reasons and the survival of patients diagnosed with $B C$ in comparison with ploidy entities are presented.

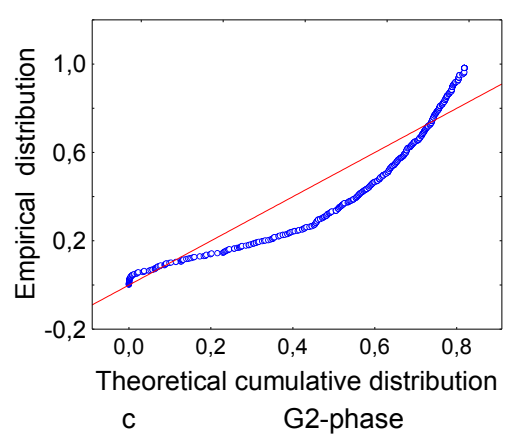

c

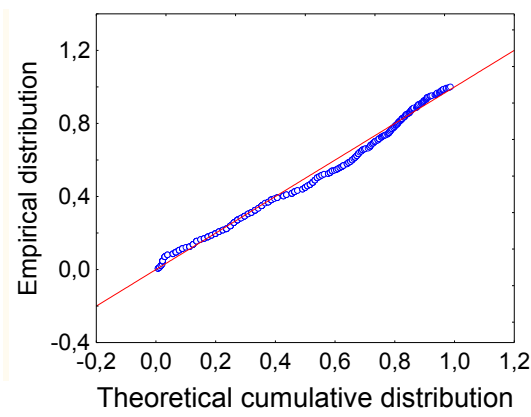

b

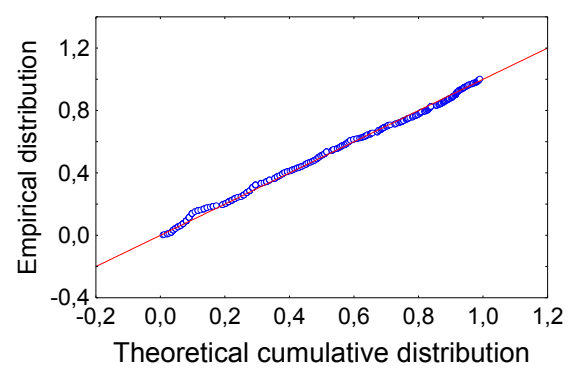

d

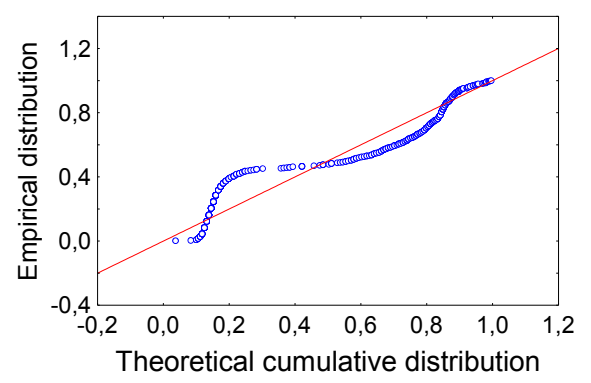

Figure 2: Probability - Probability plots in a lognormal distribution. Empirical cumulatively estimated cell cycle parameters were plotted against a theoretical cumulative distribution. A significant deviation from the theoretical curve was found for the G1-phase curve (a) and DNA index (d). Values for S- (b) and G2-phases (c) are well adjusted to the theoretical curve. 
Citation: Sennerstam RB, Strömberg JO (2013) Hyperdiploidy Tetraploidization and Genomic Instability in Breast Cancer-A Case Report Study. J Carcinogene Mutagene 4: 144. doi:10.4172/2157-2518.1000144

Page 5 of 9

\section{Genomic reorganization}

The histograms in Figure 3 showed signs of a transient tetraploidization during increased proliferative activity and genomic instability (SSI). We therefore followed the relationship between the percentages of D-, A-, and T-type tumors at each point as SSI values increased up to 40 relative units. Figure $3 \mathrm{a}$ shows a typical slow-growing tumor distribution of DI values. In Figure 4 data was collected as increasing accumulated values and presented as relative percentages of D-, A-, and T-type tumors during increasing levels of genomic instability (SSI), which reveals alteration trends. In the first
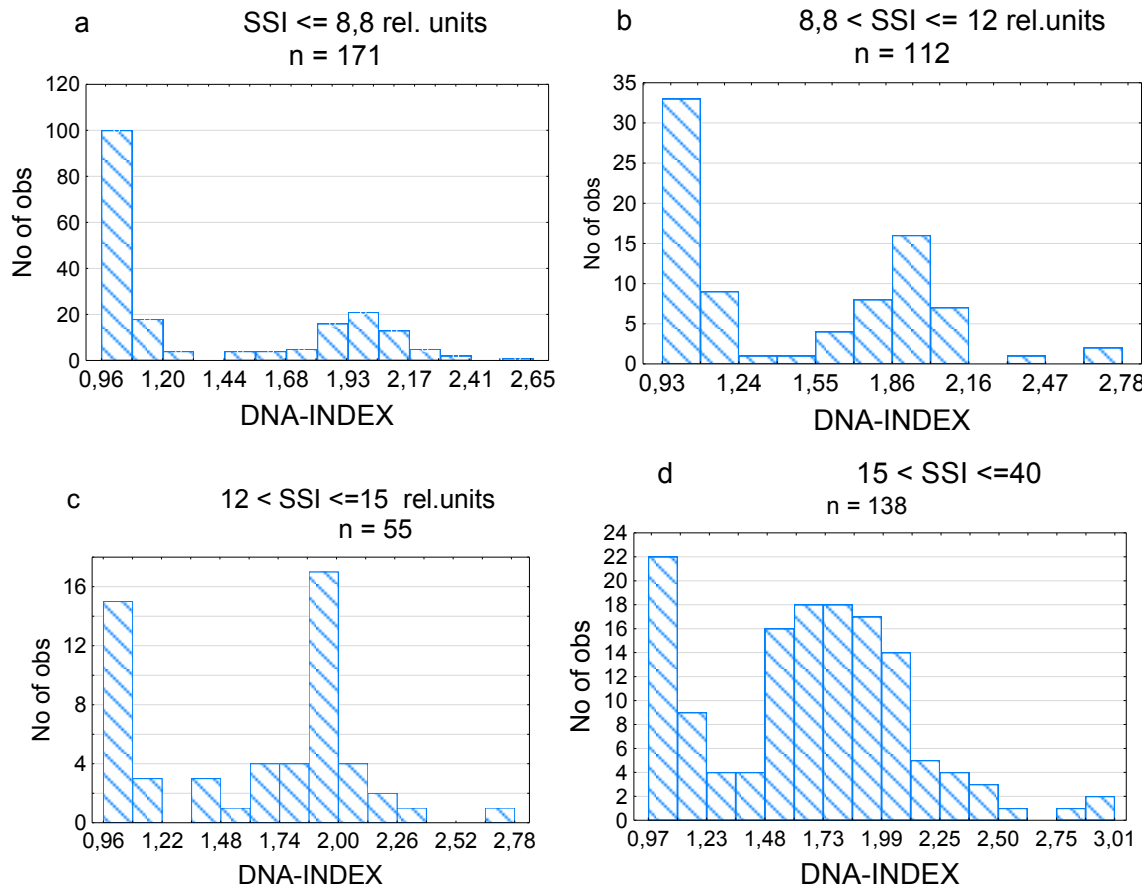

Figure 3: Distribution of DNA index histograms in four increasing SSI intervals. (a) In the SSI interval $\leq 8.8$, D-type tumors predominate and there are some T-type tumors. (b-c) After a temporary enhanced T-type population in $8.8<\mathrm{SSI} \leq 12$ and $12<\mathrm{SSI} \leq 15$, A-type tumors dominate in $15<\mathrm{SSI} \leq 40$ (d). The mean tumor size in the four SSI intervals was (in $\mathrm{mm}$ ) (a) $19.0 \pm 13.3$, (b) $20.9 \pm 15.6$, (c) $17.5 \pm 8.5$, and (d) $21.1 \pm 12.2$.
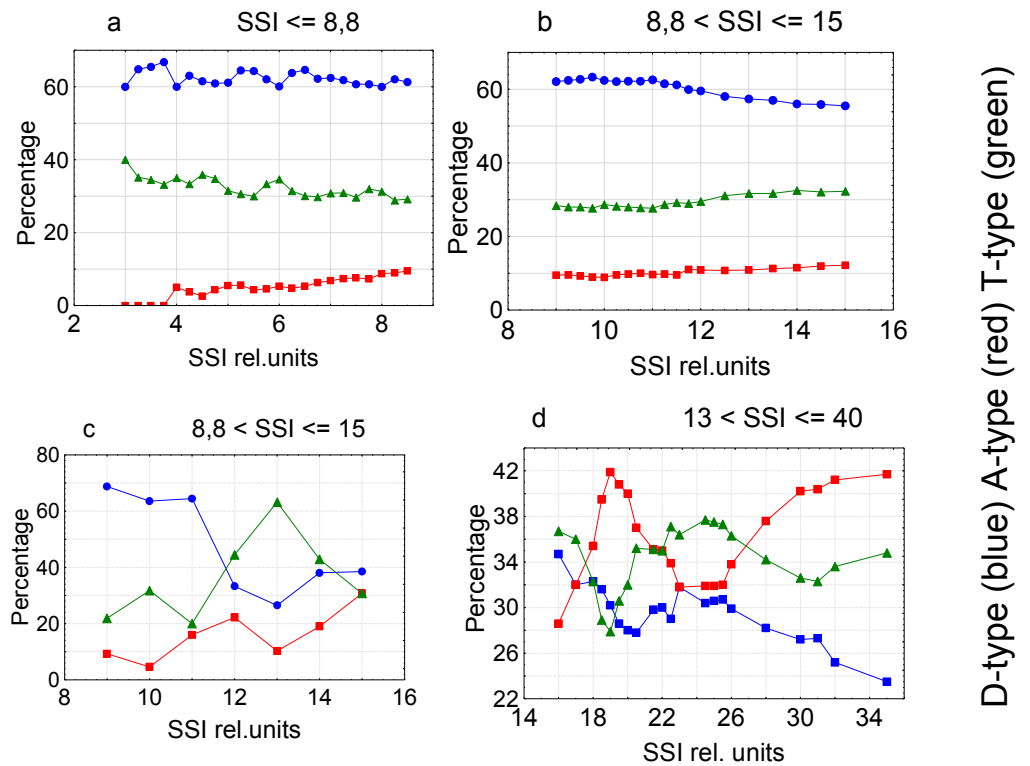

Figure 4: The percentage ratio between the three ploidy tumor entities and SSI. During three accumulatively increasing intervals of SSI values (a, b and d), mutual alterations in the percentages of D-, T-, and A-type tumors are shown. To increase the resolution, the accumulative curve in (b) was changed to values of separated intervals in (c). This reveals the tetraploid peak more clearly. 
part of the curve (Figure 4a), only D- and T-type tumors are apparent $(n=20)$, while A-type tumors appear at SSI $=4$ relative units. The curve for D-type tumors stabilizes at $60-63 \%(r=0.15, p=0.54)$. In T-type tumors (40-33\%), a negative slope was observed that was statistically significant in linear regression analysis $(\mathrm{r}=-0.78, p<0.001)$; A-type tumors $(7-10 \%)$ had a positive slope $(r=0.90, p<0.001)$. A comparison of A- and T-type tumors using linear regression analysis did result in a negative slope $(\mathrm{r}=-0.76, p<0.001)$. As the slope of each linear regression curve is not shown separately, the $r$-values are stronger than the curves appear as slopes in Figure 4a, in which three curves share the same plot of $100 \%$ (compare Figure.7).

\section{Evidence for tetraploidization}

In Figure $4 \mathrm{~b}(8.8<\mathrm{SSI} \leq 15)$, a downward slope is seen in the latter portion of the D-type curve and the T-type curve has an upward inclination. Linear regression analysis between $\mathrm{T}$ - and $\mathrm{D}$-type curves revealed a significantly negative difference $(r=-0.95, p<0.0001)$ that is in agreement with the results shown in Figures $3 b$ and $3 c$ in which a high $\mathrm{T}$-type bar appears close to $\mathrm{DI}=2.0$. To obtain a higher level of resolution, we collected data in separate intervals instead of accumulating values and found that the shift between D- and T-type curves appeared more distinct (Figure 4c): T-type tumors increased from 21.9 to $63.2 \%$ ( $p<0.001)$, D-type tumors decreased from 68.8 to $26.5 \%(p<0.001)$, and A-type tumors increased from 10.3 to $30.8 \%$ $(p<0.001)$.

Figure $4 \mathrm{~d}$ shows large disparities between the three-ploidy entities. The T-type tumors continue to decrease from their higher level in Figure 4c, A-type tumors increase to their highest level (42\%) and decline thereafter at the same time as T-type tumors increase like a new wave of tetraploidization. At higher SSI relative units, A-type tumors increased steadily (40-45\%), T-type tumors stabilized (32-34\%), and D-type tumors fell steadily to a low level of $22 \%$.

\section{Tumor size interval and ploidy reorganization}

A tumor size interval between 15.5 and $17.6 \mathrm{~mm}$ for D- and A-type tumors in the SSI interval $11<$ SSI $\leq 20$ relative units and $17.9-18-4 \mathrm{~mm}$ for T-type tumors in the SSI interval $5<$ SSI $\leq 11$ relative units (Table 3 ) was observed to correspond with the DI heterogeneity found (Figure 4). This compares the heterogeneity associated with the tumor size interval $10-20 \mathrm{~mm}$ (Figure 1). However, it appeared in subsequent increasing SSI interval comparing T-type tumors to D- and A-type tumors. In Figure $1 \mathrm{~b}$ the T-type tumors close to $\mathrm{DI}=2,0$ have a lower SSI values as compared to the transformed hypo-tetraploid A-type tumor population.

\section{Hyperdiploid D-type tumors and tetraploidization}

In D-type tumors, there was a positive correlation between tumors with Ex-G2 cells $(\mathrm{DI} \geq 2,2)$ and cells in the G2-phase $(1,8 \leq \mathrm{DI}<2,2)(\mathrm{r}=$ $0.39, \mathrm{p}<0.001 ; \mathrm{n}=202)$. This suggests that an increased level of $\mathrm{G} 2$ phase signals the presence of Ex-G2 cells, i.e., that ploidy changes occur above the G2 DI-range. We found that the highest values of Ex-G2 cells were limited to $1.01 \leq \mathrm{DI}<1.05$ within the DI range $1.00-1.20$, and that the highest percentage of Ex-G2 cells was 13\% (Figure 5). In the remaining DI interval $(1.05 \leq \mathrm{DI}<1.2)$, the highest Ex-G2 cell percentage was $2 \%$.

In a 17 -year follow-up period, death from $\mathrm{D}$-type tumors within three years of diagnosis $(n=16)$ occurred for tumors with a mean $\mathrm{DI}<1.050$ (Table 4), which is within the interval that includes advanced Ex-G2 cells (see above). These patients had higher SSI values than those who died after five years $(n=32)$ with a mean DI of 1.069 . The majority of patients with D-type tumors survived for at least 17 years $(\mathrm{n}=102)$, had a mean DI of $1.034 \pm 0.049$ and had a low SSI (Table 4). This suggests that the $\mathrm{D}$-type population involved in generating genomic instability is a relatively small population. Linear regression analysis between the months elapsed to death and DI values for all patients with D-type tumors who died was significantly positive $(\mathrm{r}=0.38, p<0.005$; $\mathrm{n}=54$ ) (Figure 6). Thus, early death from D-type BC was observed within the lower DI region $(\mathrm{DI}<1.05)$, some of which were undergoing a process of polyploidization.

\section{Hyperdiploid versus T- and A-type tumors}

To investigate the link between $\mathrm{D}$ - and T-type tumors in tetraploidization originating from a particular D-type DI interval, we calculated scatter-plots between D-type DI values and the alterations in percentages of $\mathrm{T}$ - and A-type tumors below SSI $\leq 15$ relative units

\begin{tabular}{|c|c|c|c|}
\hline & $\begin{array}{c}\text { D-type } \\
0.9 \leq \mathrm{DI}<1.2\end{array}$ & $\begin{array}{c}\text { A-type } \\
1.2 \leq \mathrm{DI}<1.8\end{array}$ & $\begin{array}{c}\text { T-type } \\
1.8 \leq \mathrm{DI}<2.2\end{array}$ \\
\hline $\mathrm{SSI} \leq 5$ & $14.6 \pm 6.4 \mathrm{n}=29$ & $24.7 \pm 13.6 \mathrm{n}=3$ & $20.6 \pm 16.6 \mathrm{n}=16$ \\
\hline $5<\mathrm{SSI} \leq 8.8$ & $19.5 \pm 14.4 \mathrm{n}=75$ & $26.2 \pm 9.5 \mathrm{n}=13$ & $18.4 \pm 11.1 \mathrm{n}=32$ \\
\hline $7<\mathrm{SSI} \leq 11$ & $20.6 \pm 17.8 \mathrm{n}=24$ & $20.2 \pm 9.5 \mathrm{n}=59$ & $17.9 \pm 9.7 \mathrm{n}=24$ \\
\hline $11<\mathrm{SSI} \leq 15$ & $16.4 \pm 7.8 \mathrm{n}=26$ & $17.6 \pm 9.0 \mathrm{n}=24$ & $22.3 \pm 13.1 \mathrm{n}=33$ \\
\hline $13<\mathrm{SSI} \leq 20$ & $15.5 \pm 7.5 \mathrm{n}=40$ & $15.5 \pm 8.1 \mathrm{n}=25$ & $21.5 \pm 9.2 \mathrm{n}=24$ \\
\hline $20<\mathrm{SSI} \leq 70$ & $23.3 \pm 10.7 \mathrm{n}=15$ & $24.3 \pm 10.7 \mathrm{n}=15$ & $20.7 \pm 8.7 \mathrm{n}=29$ \\
\hline
\end{tabular}

Table 3: Relationship between tumor size and increasing SSI according to tumor type. The three ploidy entities were investigated according to tumor size related to five SSI intervals. The values in bold are highlighting two close tumor size values for each ploidy entities found in the size interval 10 to $20 \mathrm{~mm}$ (Figure1a). For D-, and A-type tumors it appears in the SSI-interval $11<\mathrm{SSI} \leq 20$ relative units corresponding to the genomic reorganization found (Figure 3c-d and Figure 4c-d). For T-type tumors if was found at a lower level of genomic instability i.e. lower SS values $(5<\mathrm{SSI} \leq 11)($ see Table $1 \mathrm{~b})$.

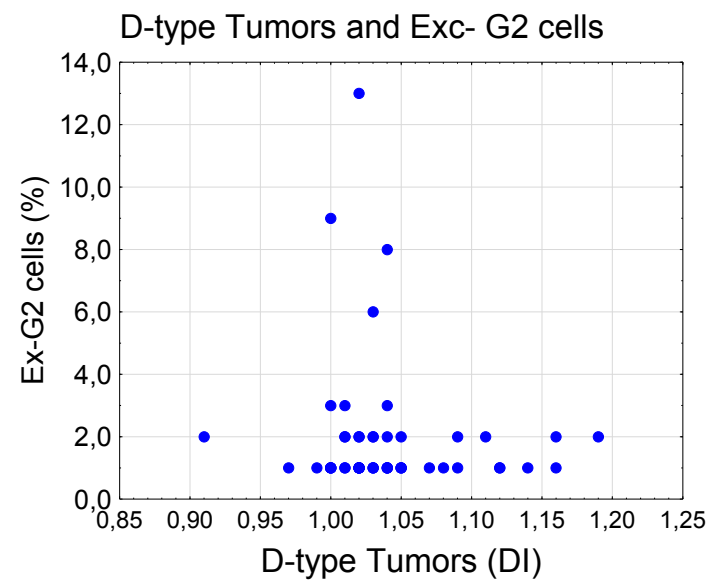

Figure 5: Relationship between D-type tumors and Ex-G2 cells. The level of Ex-G2 cells in D-type tumors showed increased values in a narrow DI interval around 1.01<DI $\leq 1.05$. Compare with Figure 7 and Table 4.

\begin{tabular}{|c|c|c|c|c|c|}
\hline & \multicolumn{4}{|c|}{ Death after number of years } & \multirow{2}{*}{ Alive } \\
\hline & $\leq 1$ & $1-3$ & $3-5$ & $5-17$ & \\
\hline $\begin{array}{c}\text { Number } \\
\text { (n) }\end{array}$ & 6 & 10 & 6 & 32 & 102 \\
\hline $\mathrm{DI}$ & $1.037 \pm 0.064$ & $1.040 \pm 0.037$ & $1.057 \pm 0.055$ & $1.069 \pm 0.049$ & $1.034 \pm 0.049$ \\
\hline SSI & $11.77 \pm 6.04$ & $14.42 \pm 9.56$ & $15.96 \pm 13.69$ & $9.23 \pm 3.97$ & $9.28 \pm 7.37$ \\
\hline
\end{tabular}

Table 4: D-type tumor relapse-free survival and death over 17 years of follow up. Shortest time elapsed to death (within 3 years) occurred for D-type tumors in the DI interval: $1.037 \leq \mathrm{DI} \leq 1.040$ ), an interval within which we found the connection to T-type and A-type tumors (Figure 7a-b) 


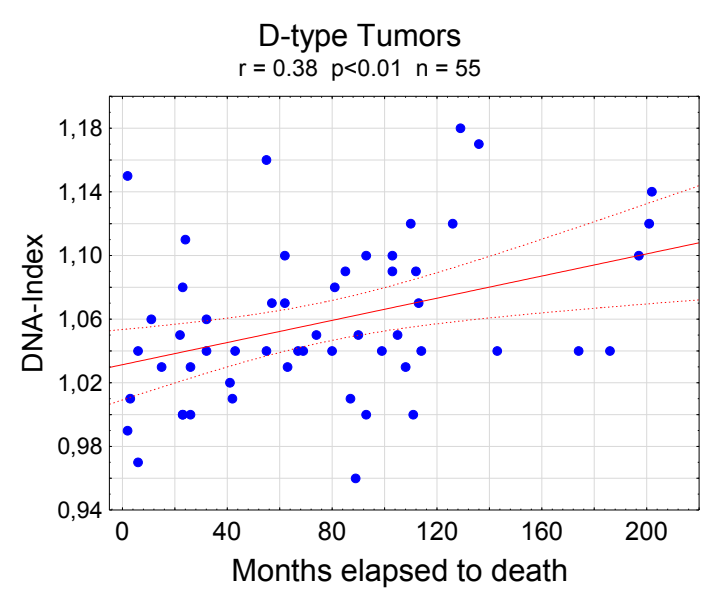

Figure 6: Time elapse to death from D-type tumors. All patients who died and were diagnosed with a D-type tumor followed a significantly positive slope in linear regression analysis $(\mathrm{r}=0.38, p<0.005 ; \mathrm{n}=54)$. Those who died within five years from diagnosis had lower DI values as compared to those who had a longer relapse-free survival period.

(Figure $7 \mathrm{a}$ and $7 \mathrm{~b}$ ), where tetraploidization occurred (Figures $3 \mathrm{c}$ and 4c). We observed a significant decrease in DI values for D-type tumors when T-type tumors increased within the DI interval: $1.020 \leq \mathrm{DI} \leq 1.044$ (Figure 7a). A-type tumors increased significantly within the same DI interval (Figure $7 \mathrm{~b} ; p<0.0001$ ).

\section{Discussion}

The evolutionary conserved phenomenon of polyploidization still occurs in humans as a reaction to various stress factors. However, it is questionable whether polyploid cells are more vulnerable to exogenous mutagenic agents such as chemicals, drugs, viruses, cigarette smoke and other carcinogenic agents. In such an environment, the division of tetraploid cells in an octoploid mitotic figure might be more vulnerable than the division of diploid cells already before genetic cell cycle checkpoints are changed.

This report documents a genomic alteration that occurs in established solid human breast tumors that have left the precancerous stages. The level of resolution was chosen to understand the "kinetics" of the significantly different D-, A-, and T-type tumors defined in Table 1. The stress placed on the tumor to change ploidy after reaching a certain size (Figures 3 and 4 ) could reflect a lack of oxygen exchange or nutrients that occurs during tumor growth in the absence of sufficient blood vessels, which might be caused by limited VEGF as well as low levels of platelet-derived endothelial cell growth factors [32]. Indeed, relapse-free survival in VEGF-rich human breast tumors was found to be significantly worse than in VEGF-poor tumors. VEGF-rich levels appeared to a limited extent in breast tumors $<2 \mathrm{~cm}$ as compared to larger tumors [22]. In the present study, it is possible that the tumor size interval $10-20 \mathrm{~mm}$ (Figure 1 and Table 3 ) in which genomic reorganizations occurred (Figures $3 \mathrm{c}, 3 \mathrm{~d}$ and $4 \mathrm{c}, 4 \mathrm{~d}$ ) might be under such anoxic stress.

The lognormal probability-probability presentation of the cell cycle data (Figure 2a-d) showed that the slope of the DI values had an obvious early upward trend. This indicates that increases in DI values do not occur slowly and continuously in the DI range $1.1 \leq \mathrm{DI}<2.2$, but rather as larger jumps of a gain of genomic material. Other cell cycle parameters as SPF and G2-phase (Figure $2 b-c$ ) were well adjusted to the lognormal curves of a growing cell population. The G1-curve had a retarded slope initially, but this increased at the end (Figure 2a) and was thought to reflect the G0 resting state early in G1-phase before the cells reenter the cell cycle at the restriction point.

In Figure 4, we followed the accumulative curves in three consecutive intervals that could be defined as low, medium, and high levels of genomic instability and growth potentials based on SSI relative units. In this instance (Figure 4a), D-type tumors dominate (60-62\%), while T-type tumors, originated as pre-diagnostic tumors in hyperplasia, dysplasia, or carcinoma in situ, have a negative slope. Either the negative relationship between $\mathrm{T}$ - and A-type tumors might reflect a transition involving a loss of chromosomal material from genomically unstable T-type tumors, or that A-type tumors might grow faster than T-type tumors. However, as this should also reduce the percentage of D-type tumors, it is more likely that T-type tumors change to hypo-Ttype tumors and become included in the A-type group of tumors in a process of selection for growth advantage. The considerable increase in A-type tumors at the beginning of the last SSI interval (Figure $4 \mathrm{~d}$ ) might reflect such an alteration. Following the rest of the last SSI interval, a new wave of tetraploidization of T-type tumors overrides the curve of the A-type tumors (Figure 4d). Finally, the A-type tumors increase to dominate, while T-type tumors stabilize around $34 \%$. This alteration in the frequency of aneuploid tumors increased death rate in BC significantly (Figure 3d). The accumulative curves in Figure $4 \mathrm{~d}$ represent a turbulent reorganization of the genome in a tumor size interval between 10 to $20 \mathrm{~mm}$ (Table 3). The concept that increases in SSI values reflect a development from tetraploid tumors towards the hypotetraploid region is supported by the significant negative slope in the $\mathrm{DI}$ region $1.5<\mathrm{DI}<2.2$ for $\mathrm{T}$ - to hypo-T-type tumors in Figure $1 \mathrm{~b}$.

In the D-type population we observed that most Exc-G2 cells were located in the same narrow D-type DI-interval (Figure 5) as where we found the connection between D-, to A-, and to T-type tumors in two dimensional linear regression analysis (Figure 7). In Table 4 a small group of $\mathrm{D}$-type patients with $\mathrm{DI}<1.044$ died within 3 years. We draw the conclusion that these tumors either have two options, tetraplodization or die soon after diagnosis as D-type tumors.
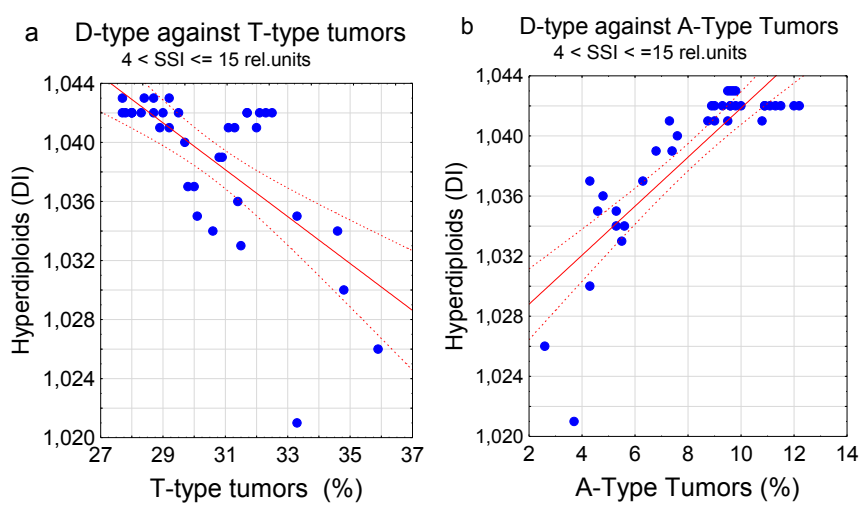

Figure 7: Relationship between DNA-Index of D-type tumors and percentages of T-, and A-type tumors. Linear regression analysis was used to assess the relation between D-type tumors and T- (a) or A-type (b) tumors within an SSI interval $4<S S I \leq 15$ relative units. A strong significant negative correlation appeared between decreasing D-type DI values of hyperdiploid tumors $(1.020<\mathrm{DI} \leq 1.044)$ and increasing percentages of T-type tumors $(r=$ $-0.68, p<0.0001 ; \mathrm{n}=37$ ). A similar but positive correlation appeared regarding the percentage of A-type tumors $(r=0.84, p<0.0001 ; n=37)$. 
Citation: Sennerstam RB, Strömberg JO (2013) Hyperdiploidy Tetraploidization and Genomic Instability in Breast Cancer-A Case Report Study. J Carcinogene Mutagene 4: 144. doi:10.4172/2157-2518.1000144

Page 8 of 9

A recent study reported that clusters of amplifications in narrow peaks of single chromosomal arms among pseudodiploid tumors were of great importance for tumor survival [33]. Our observed data of D-type tumors in Table 4 supports this theory.

The positive correlation between D-type tumors DI values and A-type tumors (Figure 7b) includes the A-type tumors in the three step sequence of increasing genomic instability suggesting that hyperdiploid cells pass over to tetraploid cells losing genetic material generating aneuploid cells most probably in a selection for growth advantage. The stepwise increase in tumor size from D- to T- and finally to A-type tumors shown in Table 1 support the theory of T-type tumors as an intermediate stage.

\section{Conclusions}

The phenomenon of polyploidization is not limited to occur only during tumor progression. Tetraploidization can be observed during human cervical, colon and lever infections and in precancerous atypia as well as aneuploidy in ulcerative colitis proceeding with a decade cancer development. However, during early tumor development of epithelial cells, tetraploidization appears as an intermediate state and subsequently turns over to aneuploidy during loss of genomic materials. In this report we show that when breast tumors reach a size to be detected D-, T-, and some A-type tumors can already have been established. Furthermore, in the breast cancer sample analyzed we found a second period of tetraploidization and a turn over to aneuploidy in a certain tumor size interval between 10 to $20 \mathrm{~mm}$. Finally we found evidence for the tetraploidy to be recruited from a narrow D-type DI interval, where D-type tumors either die early after diagnosis or being transformed to polyploid tumors.

\section{Competing Interests}

This report does not include the use of any commercial products with connections to the authors. The staining techniques used have been established since more than 40 years. There is no way to get economical benefits from a publication of this article.

\section{Acknowlegements}

We are thankful to Professor Gert Auer for offering ongoing fruitful discussions concerning tumor progression. Language editing done by Edanz Group Global Ldt. Support by Karolinska Institutes Fund.

\section{Reference}

1. Otto SP (2007) The evolutionary consequences of polyploidy. Cell 131: 452 462

2. Lee HO, Davidson JM, Duronio RJ (2009) Endoreplication: polyploidy with purpose. Genes Dev 23: 2461-2477.

3. Rochlin K, Yu S, Roy S, Baylies MK (2010) Myoblast fusion: when it takes more to make one. Dev Biol 341: 66-83.

4. Duelli D, Lazebnik $Y(2007)$ Cell-to-cell fusion as a link between viruses and cancer. Nat Rev Cancer 7: 968-976.

5. Olaharski AJ, Sotelo R, Solorza-Luna G, Gonsebatt ME, Guzman P, et al. (2006) Tetraploidy and chromosomal instability are early events during cervical carcinogenesis. Carcinogenesis 27: 337-343.

6. Levine DS, Sanchez CA, Rabinovitch PS, Reid BJ (1991) Formation of the tetraploid intermediate is associated with the development of cells with more than four centrioles in the elastase-simian virus 40 tumor antigen transgenic mouse model of pancreatic cancer. Proc Natl Acad Sci U S A 88: 6427-6431.

7. Kaufmann WK, Levedakou EN, Grady HL, Paules RS, Stein GH (1995) Attenuation of $\mathrm{G} 2$ checkpoint function precedes human cell immortalization. Cancer Res 55: 7-11.

8. Andreassen PR, Martineau SN, Margolis RL (1996) Chemical induction of mitotic checkpoint override in mammalian cells results in aneuploidy following a transient tetraploid state. Mutat Res 372: 181-194.

9. Storchova Z, Pellman D (2004) From polyploidy to aneuploidy, genome instability and cancer. Nat Rev Mol Cell Biol 5: 45-54

10. Fujiwara T, Bandi M, Nitta M, Ivanova EV, Bronson TR (2005) Cytogenesis failure generating tetraploids promotes tumorigenesis in P53-null cells. Nature 437: 1043-1047.

11. Ganem NJ, Storchova Z, Pellman D (2007) Tetraploidy, aneuploidy and cancer Curr Opin Genet Dev 17: 157-162.

12. Vitale I, Galluzzi L, Senovilla L, Criollo A, Jemaà M, et al. (2011) Illicit survival of cancer cells during polyploidization and depolyploidization. Cell Death Differ 18: $1403-1413$.

13. Ornitz DM, Hammer RE, Messing A, Palmiter RD, Brinster RL (1987) Pancreatic neoplasia induced by SV40 T-antigen expression in acinar cells of transgenic mice. Science 238: 188-193.

14. Kalliomäki TM, McCallum G, Wells PG, Hill RP (2009) Progression and metastasis in a transgenic mouse breast cancer model: effects of exposure to in vivo hypoxia. Cancer Lett 282: 98-108.

15. Bristow RG, Hill RP (2008) Hypoxia and metabolism. Hypoxia, DNA repair and genetic instability. Nat Rev Cancer 8: 180-192.

16. Rofstad EK, Johnsen NM, Lyng H (1996) Hypoxia-induced tetraploidisation of a diploid human melanoma cell line in vitro. Br J Cancer Suppl 27: S136-139.

17. Brown NS, Bicknell R (2001) Hypoxia and oxidative stress in breast cancer Oxidative stress: its effects on the growth, metastatic potential and response to therapy of breast cancer. Breast Cancer Res 3: 323-327.

18. Chi JT, Wang Z, Nuyten DS, Rodriguez EH, Schaner ME, et al. (2006) Gene expression programs in response to hypoxia: cell type specificity and prognostic significance in human cancers. PLoS Med 3: e47.

19. Huang LE, Bindra RS, Glazer PM, Harris AL (2007) Hypoxia-induced genetic instability--a calculated mechanism underlying tumor progression. J Mol Med (Berl) 85: 139-148

20. Chen JL, Lucas JE, Schroeder T, Mori S, Wu J, et al. (2008) The genomic analysis of lactic acidosis and acidosis response in human cancers. PLoS Genet 4: e1000293.

21. Yau C, Benz CC (2008) Genes responsive to both oxidant stress and loss of estrogen receptor function identify a poor prognosis group of estrogen receptor positive primary breast cancers. Breast Cancer Res 10: R61.

22. Toi M, Hoshina S, Takayanagi T, Tominaga T (1994) Association of vascula endothelial growth factor expression with tumor angiogenesis and with early relapse in primary breast cancer. Jpn J Cancer Res 85: 1045-1049.

23. Blegen H, Ghadimi BM, Jauho A, Zetterberg A, Eriksson E, et al. (2001) Genetic instability promotes the acquisition of chromosomal imbalances in T1b and T1c breast adenocarcinomas. Anal Cell Pathol 22: 123-131.

24. Skyldberg B, Fujioka K, Hellström AC, Sylvén L, Moberger B, et al. (2001) Human papillomavirus infection, centrosome aberration, and genetic stability in cervical lesions. Mod Pathol 14: 279-284.

25. Eriksson ET, Schimmelpenning H, Aspenblad U, Zetterberg A, Auer GU (1994) Immunohistochemical expression of the mutant p53 protein and nuclear DNA content during the transition from benign to malignant breast disease. Hum Pathol 25: 1228-1233.

26. Gerling M, Nousiainen K, Hautaniemi S, Krüger S, Fritzsche B, et al. (2013) Aneuploidy-associated gene expression signatures characterize malignant transformation in ulcerative colitis. Inflamm Bowel Dis 19: 691-703.

27. Schimmelpenning $\mathrm{H}$, Eriksson ET, Zetterberg A, Auer GU (1994) Association of immunohistochemical p53 tumor suppressor gene protein overexpression with prognosis in highly proliferative human mammary adenocarcinomas. World $J$ Surg 18: 827-832.

28. Fukasawa K (2005) Centrosome amplification, chromosome instability and cancer development. Cancer Lett 230: 6-19.

29. Duesberg R, Li R, Fabarius A, Hehlmann R (2006) Aneuploidy and cancer: from correlation to causation. Dittmar T, Zaenker KS, Schmidt A. (eds): Infection and Inflammation: Impacts on Oncogenesis. Contrib Microbiol. vol 13. 16-44.

30. Forsslund G, Esposti PL, Nilsson B, Zetterberg A (1992) The prognostic 
Citation: Sennerstam RB, Strömberg JO (2013) Hyperdiploidy Tetraploidization and Genomic Instability in Breast Cancer-A Case Report Study. J Carcinogene Mutagene 4: 144. doi:10.4172/2157-2518.1000144

Page 9 of 9

significance of nuclear DNA content in prostatic carcinoma. Cancer 69: 1432 1439

31. Kronenwett U, Huwendiek S, Ostring C, Portwood N, Roblick UJ, et al. (2004) Improved grading of breast adenocarcinomas based on genomic instability. Cancer Res 64: 904-909.

32. Toi M, Inada K, Hoshina S, Suzuki H, Kondo S, et al. (1995) Vascular endothelial growth factor and platelet-derived endothelial cell growth factor are frequently coexpressed in highly vascularized human breast cancer. Clin Cancer Res 1: 961-964.

33. Hicks J, Krasnitz A, Lakshmi B, Navin NE, Riggs M, et al. (2006) Novel patterns of genome rearrangement and their association with survival in breast cancer. Genome Res 16: 1465-1479. 